\title{
Quantitative assessment of aerospace materials by active thermography techniques
}

\author{
by C. Ibarra-Castanedo*, E. Grinzato**, S. Marinetti**, P. Bison**, N. Avdelidis*, M. Grenier*, J-M. \\ Piau*, A. Bendada* and X. Maldague*
}

${ }^{*}$ Computer Vision and Systems Laboratory, Department of Electrical and Computer Engineering, Université Laval, Quebec City, Canada G1K 7P4, Phone: +1(418) 656-2962, Fax:+1(418) 656-3594, E-mail: \{lbarraC, Grenier, Bendada,MaldagX\}@gel.ulaval.ca,Avdel@mail.ntua.gr

**Istituto per le Tecnologie della Costruzione - Consiglio Nazionale delle Ricerche (ITC-CNR), Corso Stati Uniti, 435127, Padova, Italy. Phone: +39(049)829-5722, Fax: +39(049)829-5728, E-mail: \{Ermanno.Grinzato, Sergio.Marinetti,Paolo.Bison\}@itc.cnr.it

\begin{abstract}
The inspection of aerospace materials such as composites, honeycomb structures, ceramics and GLARE has been the subject of many studies in the NonDestructive Testing and Evaluation (NDT\&E) community. Active thermography techniques have shown to be an interesting alternative or complementary to other classical NDT\&E techniques. Nevertheless, the different techniques perform differently depending on the application. This study is part of a collaboration project between Laval University of Quebec, Canada and the ITC of Padua Italy in order to investigate the ability of pulsed and modulated thermography to inspect aerospace materials through optical, mechanical or electromagnetically induced excitation.
\end{abstract}

\section{Introduction}

Infrared thermography is an NDT technique allowing fast inspection of large surfaces [1]. There are different techniques depending on the stimulation source, basically: pulsed, step or modulated. The specimen is stimulated with an energy source, which can be of many types, such as optical, mechanical or electromagnetic. Optical energy is normally delivered externally, i.e. heat is produced at the surface of the specimen from where it travels trough the specimen to the subsurface anomally (defect) and back to the surface. Mechanical energy on the other hand, can be considered as an internal way of stimulation, since heat is generated at the defect interface and then travels to the surface. In electromagnetic excitation, Eddy currents are externally induced to the material (conductor), and heat is produced internally from the circulation of this currents in the material. Moreover, energy may be delivered in transitory or steady state regime. For instance, pulsed thermography, which is typically performed using a heat pulse of a few milliseconds can be considered as an optical-external technique in transitory regime. A thermal map of the surface or a thermogram is recorded at regular time intervals. Every material responds differently depending on the way it has been stimulated. For instance, mechanical excitation can be used to easily detect micro-cracks on or under the surface of a conductor specimen, whilst this type of defect would be very difficult to detect by optical excitation. On the contrary, optical techniques provide in general a much better defect resolution than mechanical excitation in the case of delaminations on composites or sandwich structures, although mechanical excitation provides information on deeper delaminations than optical testing. Electromagnetic excitation is a relatively new field of investigation in active thermography. As in conventional Eddy current testing, Eddy current thermography is limited to the inspection of conductor materials. Some studies have shown the potential of Eddy current thermography for the inspection of conductor materials that traditionally poses a problem to classical NDT\&E techniques, such as GLARE.

The following section provides two examples of application of specific active thermography techniques for a particular material.

\section{Experimental results}

It was possible to view the impact damage on the surface, especially in the case of the thinner composite panels such as specimen GLARE006 (Figure 1, top). Furthermore, even in the case of the thicker samples as specimen GLARE007 (Figure 1, bottom) the defected areas, created by the impact damage testing, could be picked up by pulsed thermography as can be seen in Figure 1a. Processing results by pulsed phase thermography [2] improve defect contrast and provides an indication about the extent of the internal damage. In accordance with the phase probing properties [3], the phasegrams at $1 \mathrm{~Hz}$ (Figure 1b) provide information about deeper features than the phasegrams at $0.15 \mathrm{~Hz}$. It can be observed from these results that the extend of damage is greater, for the case of a thin plate subjected to a high energy impact $(8 \mathrm{~J})$ than for a thick plate subjected to a lower impact energy $(4 \mathrm{~J})$, as expected.

C-scan ultrasounds is routinely used in the assessment of GLARE panels. However, the inspection is lengthy with the component being removed from the aircraft as shown in Figure 2. Pulsed thermography results (Figure 2, bottom) demonstrate that is possible to detect the lack of material down to a certain depth. Only two of the tree fabricated defects can be seen in either front or back side testing. The conventional Eddy current result show perfectly the tree defects, which let us to conclude that Eddy current thermography could provide comparable results with a fully portable equipment for the inspection in situ. Experimental results will be presented on the final version of this paper and during the conference. 

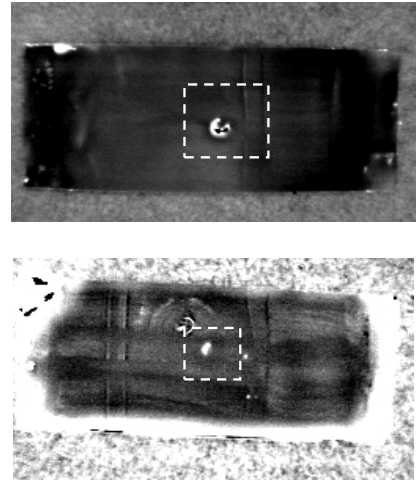

(a)
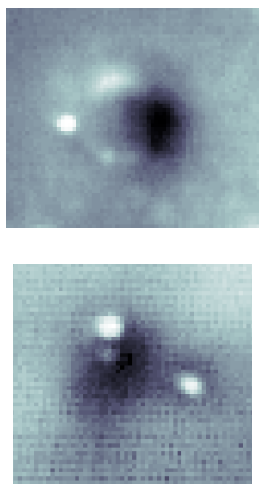

(b)
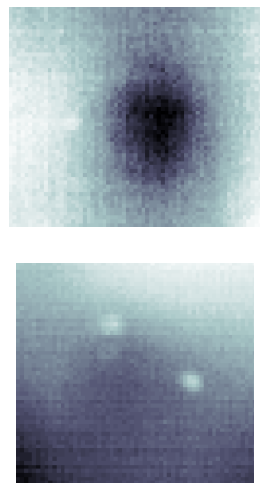

(c)

Figure 1. Results for specimens GLARE006 (top) and GLARE007 (bottom) after impact damage testing using 8 and $4 \mathrm{~J}$, respectively: (a) thermogram, and the corresponding phasegrams of the cropped portion obtained by PPT at $f=($ b) 1 and (c) $0.15 \mathrm{~Hz}$.

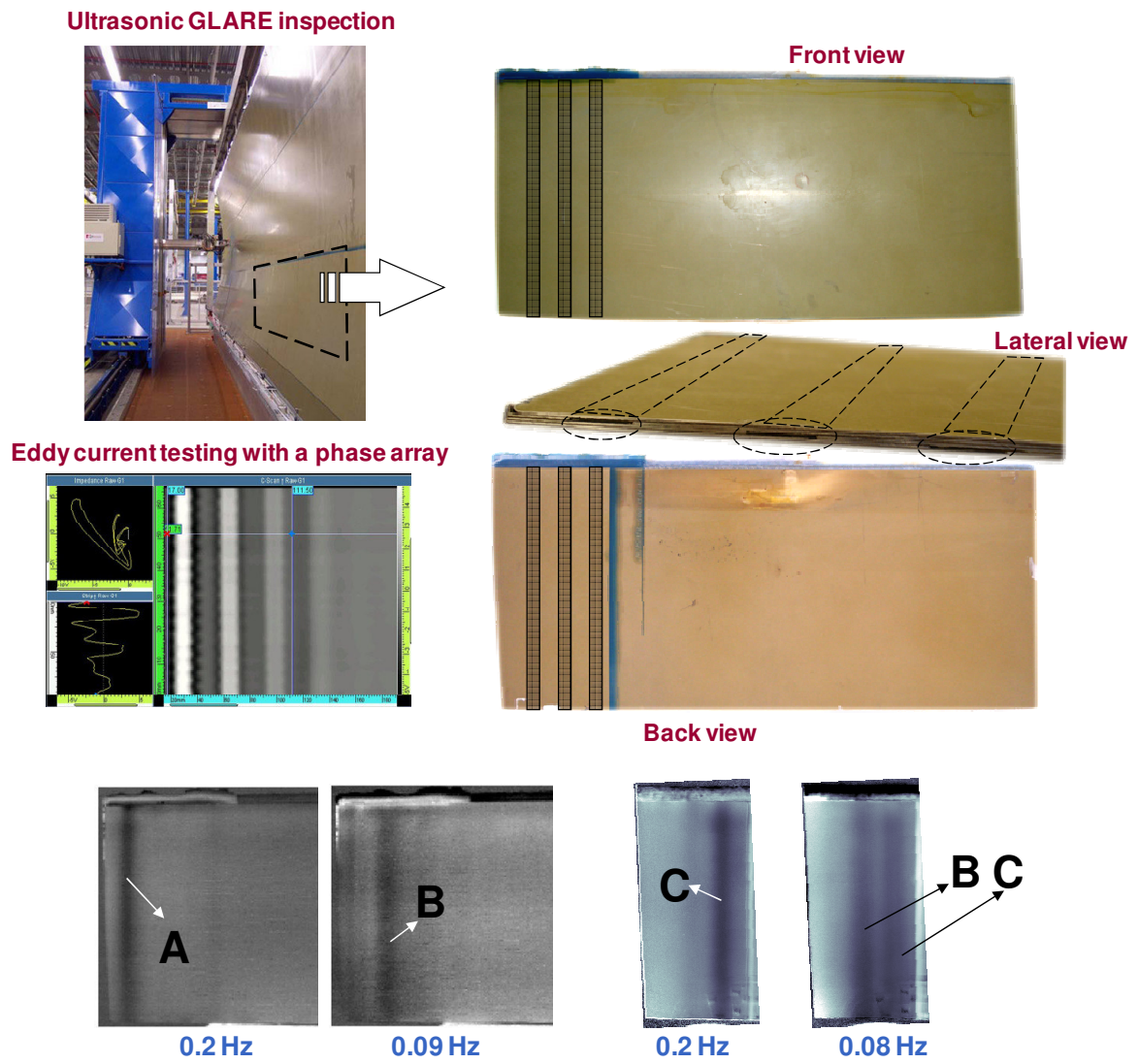

Figure 2. GLARE inspection by ultrasonic testing (top left), GLARE section with fabricated defects (right), and results on the GLARE section by phase array Eddy current testing (bottom left).

\section{REFERENCES}

[1] X. P. V. Maldague, Theory and Practice of Infrared Technology for NonDestructive Testing, John WileyInterscience, 684 p., 2001.

[2] X. P. Maldague, S. Marinetti, Pulse Phase Infrared Thermography, J. Appl. Phys., 79(5):2694-2698, 1996.

[3] C. Ibarra-Castanedo and X. Maldague. "Pulsed Phase Thermography Reviewed," QIRT J., 1(1):47-70, 2004. 